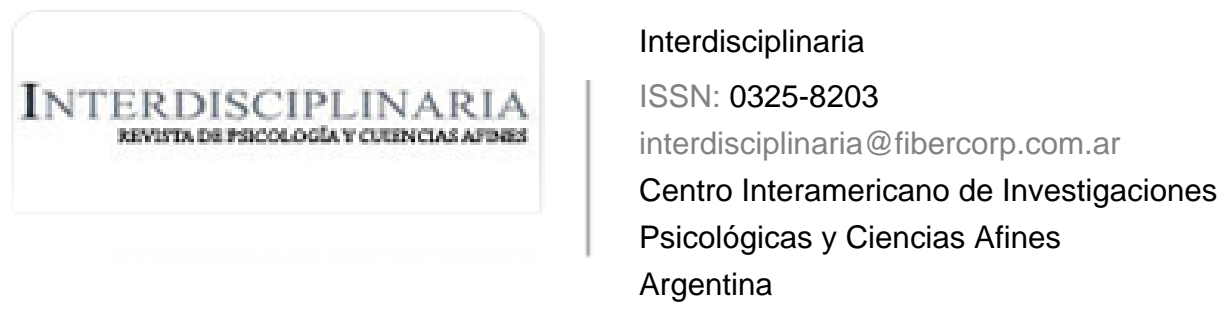

DANERI, MARÍA FLORENCIA; MUZIO, RUBÉN NÉSTOR

EVOLUCIÓN DE FENÓMENOS BÁSICOS DE APRENDIZAJE EN TAREAS DE MEMORIA ESPACIAL: BLOQUEO, ENSOMBRECIMIENTO E INHIBICIÓN LATENTE EN ANFIBIOS

Interdisciplinaria, vol. 32, núm. 2, 2015, pp. 275-288

Centro Interamericano de Investigaciones Psicológicas y Ciencias Afines

Buenos Aires, Argentina

Disponible en: http://www.redalyc.org/articulo.oa?id=18043528005

Cómo citar el artículo

- Número completo

- Más información del artículo

- Página de la revista en redalyc.org

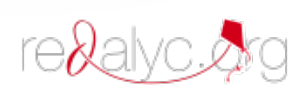

Sistema de Información Científica

Red de Revistas Científicas de América Latina, el Caribe, España y Portugal Proyecto académico sin fines de lucro, desarrollado bajo la iniciativa de acceso abierto 


\title{
EvoluCIÓN DE FENÓMENOS BÁSICOS DE APRENDIZAJE EN TAREAS DE MEMORIA ESPACIAL: BLOQUEO, ENSOMBRECIMIENTO E INHIBICIÓN LATENTE EN ANFIBIOS*
}

\section{EVOLUTION OF BASIC LEARNING PHENOMENA IN SPATIAL MEMORY TASKS: BLOCKING, OVERSHADOWING AND LATENT INHIBITION IN AMPHIBIANS}

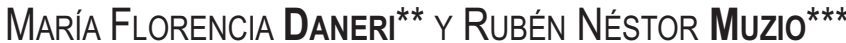

\author{
*Trabajo financiado con el subsidio UBACYT-P052 de la Universidad de Buenos Aires (UBA) y el PIP 3196 del \\ Consejo Nacional de Investigaciones Científicas y Técnicas (CONICET). \\ ${ }^{*}$ Doctora en Ciencias Biológicas. Profesora investigadora de la Facultad de Psicología de la Universidad de Buenos \\ Aires (UBA). E-Mail: flordaneri@yahoo.com \\ ***Doctor en Ciencias Biológicas. Miembro de la Carrera del Investigador Científico del Consejo Nacional de \\ Investigaciones Científicas y Técnicas (CONICET), Profesor Asociado Regular de la Facultad de Psicología de la \\ Universidad de Buenos Aires (UBA), Director del Grupo de Aprendizaje y Cognición Comparada del Laboratorio de \\ Biología del Comportamiento (IBYME) y Director de los subsidios UBACYT P052 y PIP 3196 del Consejo Nacional de \\ Investigaciones Científicas y Técnicas (CONICET). E-Mail: rnmuzio@gmail.com \\ Grupo de Aprendizaje y Cognición Comparada. Laboratorio de Biología del Comportamiento. Instituto de Biología y \\ Medicina Experimental (IBYME). Vuelta de Obligado 2490 \\ (C1428ADN) Ciudad Autónoma de Buenos Aires - República Argentina.
}

\section{REsUmen}

En este artículo se describen desde una perspectiva comparativa los fenómenos de bloqueo, ensombrecimiento e inhibición latente, enfatizando su presencia en tareas de aprendizaje espacial. Estos fenómenos de aprendizaje, ampliamente observados en otras clases de vertebrados e invertebrados, han sido recientemente descriptos por primera vez en anfibios, un grupo de vertebrados con un cerebro filogenéticamente antiguo. Tomando como modelo al sapo terrestre Rhinella arenarum, se revisarán los tres fenómenos de aprendizaje asociativo mencionados en una situación de aprendizaje espacial: (1) bloqueo entre claves visuales asociadas a una meta, (2) ensombrecimiento de una clave visual lejana por la presencia de una clave cercana y (3) inhibición latente debida a la pre-exposición a una clave visual. Todos los entrenamientos se llevaron a cabo en una arena circular de color blanco, uti- lizando agua como recompensa. Dentro de la arena, se distribuyeron cuatro piletas de acrílico en forma de cruz contra las paredes laterales (sólo una tenía acceso a la recompensa). En las paredes interiores de la arena circular se colocaron varias señales visuales para guiar a los animales. Los resultados obtenidos en sapos indican que estos fenómenos, observados previamente en aves y mamíferos, también se encuentran en este grupo (utilizando un paradigma de aprendizaje espacial con claves visuales cercanas y lejanas). Este primer registro en anfibios sugiere que los mecanismos biológicos de estos fenómenos de aprendizaje han surgido muy tempranamente en el curso de la evolución de los vertebrados totalmente terrestres y que los mismos han sido fuertemente conservados. El análisis comparado de estos hallazgos contribuirá a mejorar el entendimiento de los mecanismos biológicos que subyacen al aprendizaje espacial, en busca de patrones funcionales comunes con otras clases de verte- 
brados y potencialmente presentes en un ancestro común.

Palabras clave: Bloqueo; Ensombrecimiento; Inhibición latente; Aprendizaje espacial; Anfibios.

\section{ABSTRACT}

This article describes blocking, overshadowing and latent inhibition phenomena from a comparative perspective, emphasizing their presence in spatial learning tasks. These learning phenomena, previously observed in other vertebrates and invertebrates classes, have been recently reported for the first time in amphibians, a vertebrate group with a phylogenetically ancient brain. We use the terrestrial toad Rhinella arenarum as animal model to analyze the three mentioned associative phenomena in a spatial learning situation: (1) blocking between visual cues associated to a goal, (2) overshadowing of a distant visual cue by the presence of a nearby cue, and (3) latent inhibition generated by the pre-exposure to a visual cue. All trainings were conducted in a white circular arena, using water as reward. Inside the arena, four green acrylic container were distributed in a cross shape against the side walls (only one with access to reward). On the inner walls of the circular arena they were placed several visual cues for guiding animals. In all studies described in this article toads were partially dehydrated to motivate them to search for water. In the blocking situation, experimental animals had the rewarded container signaled by a visual cue on the wall above the container. In the second phase of training other visual cue was added. The results revealed that in these animals the prior training with only one of the visual cues blocked the association of the reward with the other cue, when both cues were then presented simultaneously to indicate the position of reward. In the overshadowing situation, experimental animals had from the beginning the rewarded container signaled by two visual cues on the wall, one to $10 \mathrm{~cm}$ right (named near cue), and the other placed approximately $70 \mathrm{~cm}$ to the left (between the adjacent pool and the opposite, named far cue). The results indicated that the location of a visual cue located away from reward was overshadowed by the presence of a nearby cue. Finally, in the situation of latent inhibition, animals of pre-exposed group had five previous training sessions, where a visual cue was presented without reward. Then, when in the subsequent training this visual cue signaled the reward, animals pre-exposed needed more sessions to reach the asymptote of learning compared to other non-pre-exposed animals. Therefore, the preexposure to the visual cue (i.e., unreinforced exposure to the stimulus) significantly retarded the acquisition, delaying the association of this cue with the reward. Taking into account that these phenomena have been observed previously in birds and mammals, this first record in amphibians (using a spatial learning paradigm with near and far visual cues) suggests that biological mechanisms of these learning phenomena have emerged very early in the course of the evolution of fully terrestrial vertebrates and that they have been strongly preserved. With regard to the underlying neural substrates, the relationship of the hippocampal formation with the processes of learning and spatial memory is a constant in all vertebrate species studied. Analyzing the particular case of amphibians, compared with other groups of tetrapods, their telencephalon has a simple organization (in the evolutionary sense non-derived). In this aspect, the medial pallium, region in the dorsomedial quadrant of the hemisphere, is considered homologous to the mammalian hippocampal formation (based on their topographic position, its interconnections with other telencephalic areas and neurohistochemical data). Heretofore, the dependence of spatial learning with the functioning of the medial cortex was thought to be a primitive character of amniotes. However, this feature can now be extended to the group of amphibians, as recently has been observed that the lesion of the medial pallium impairs spatial learning. Thus, the evidence collected until this moment in amphibians suggests that this relationship may have emerged earlier than previously thought.

On the whole, the comparative analysis of these findings will contribute to a better understanding of the biological mechanisms underlying spatial learning, thereby looking for common functional patterns with other vertebrate classes, potentially present in a common ancestor.

Key words: Blocking; Overshadowing; Latent inhibition; Spatial learning; Amphibians. 


\section{INTRODUCCIÓN}

Un proceso de aprendizaje está generalmente acompañado por un cambio gradual en el comportamiento. Cuando ocurre este cambio, se asume como una consecuencia de la actividad nerviosa y su estudio se usa para probar si el funcionamiento del cerebro del individuo ha cambiado a través de la experiencia (Lüddecke, 2003). Desde la perspectiva de la Psicología Comparada, un comportamiento aprendido debe ser analizado bajo condiciones que permitan establecer su función en relación con las demandas del ambiente, y para determinar su valor adaptativo, éste debe ser asociado con la historia natural de la especie en estudio (Lüddecke, 2003; Papini, 2002, 2009).

Al analizar ciertas habilidades cognitivas, como la posibilidad de orientación en el espacio mediante el aprendizaje espacial, surge la incógnita de cómo ha aparecido esta capacidad en términos evolutivos. Una de las maneras de intentar resolver este enigma es realizando un análisis comparado de la conducta en grupos filogenéticamente antiguos tales como anfibios, reptiles y aves (Muzio, 2013; Papini, 2002, 2009).

Si se analiza la capacidad de aprendizaje espacial en vertebrados en general, varios estudios han demostrado que los mamíferos y las aves pueden utilizar diferentes estrategias para la orientación en el espacio, basadas en múltiples sistemas de aprendizaje con el objetivo de orientarse y luego retornar al propio territorio-hogar (López, Broglio, Rodríguez, Thinus-Blanc \& Salas, 1999). A pesar de la importancia de los estudios comparativos para comprender la estructura y el funcionamiento del aprendizaje espacial y los sistemas de memoria en vertebrados (Sherry \& Schacter, 1987), sólo unos pocos han analizado los mecanismos del aprendizaje espacial en vertebrados filogenéticamente más antiguos tales como peces (López et al., 1999), tortugas (Grisham \& Powers, 1990; López, Vargas, Gómez \& Salas, 2003) y lagartijas (Day, Ismail \& Wilczynski, 2003), siendo muy pocos los que lo han hecho en anfibios (Daneri, Casanave \& Muzio, 2011, 2015a; Muzio, 2013).
Los estudios naturalistas de la conducta migratoria en anfibios (Freeland \& Martin, 1985; Gallardo, 1987; Tunner, 1992) parecerían sugerir que pueden utilizar diferentes estrategias espaciales, similares a aquellas observadas en mamíferos. Los movimientos de los anfibios están generalmente guiados por una conducta de orientación destinada a elegir la distancia más corta hacia la meta a fin de minimizar el riesgo de predación y deshidratación. Individuos de muchas especies de anfibios desplazados de su territorio son capaces de relocalizar sitios conocidos utilizando una variedad de claves direccionales y mecanismos de orientación (Sinsch, 1987). La información sensorial obtenida de una potencial clave está jerarquizada y es específica para cada especie, e incluso para cada población; esto permite maximizar el uso de la información disponible en los ambientes naturales (Sinsch, 2006).

Para estudiar los mecanismos subyacentes al aprendizaje espacial en anfibios en un marco comparativo, se han revisado tres fenómenos básicos de aprendizaje: el bloqueo, el ensombrecimiento y la inhibición latente entre claves visuales. La presencia de estos fenómenos ha sido ampliamente demostrada en varios grupos de vertebrados, incluyendo mamíferos, y algunos recientemente observados en anfibios en nuestro laboratorio (Daneri, Casanave \& Muzio, 2015b; Daneri \& Muzio, 2013). Los anfibios representan un grupo de transición entre el modo de vida acuático y terrestre entre los vertebrados, por ello su estudio es central para comprender los mecanismos evolutivos asociados a la colonización de la tierra y todas las adaptaciones vinculadas en este proceso (Muzio, 2013). La información obtenida en este tipo de análisis comparado es entonces clave para el estudio de la evolución de estos fenómenos, ya que analizar su presencia en anfibios permite sentar las bases para luego describir los mecanismos neurales que subyacen a estos aprendizajes. El objetivo final es la búsqueda de patrones funcionales comunes con otras clases de vertebrados, potencialmente presentes en un ancestro común $(\mathrm{Mu}-$ zio, 2013). 


\section{BLOQUEO Y ENSOMBRECIMIENTO}

Se ha observado que el entrenamiento previo con una sola clave (Estímulo Condicionado - EC1) interfiere la asociación del refuerzo con otra clave (EC2) cuando en una segunda fase de entrenamiento, ambas claves $(\mathrm{EC} 1+\mathrm{EC} 2)$ son presentadas simultáneamente señalando la posición del refuerzo (Estímulo Incondicionado - EI). Este fenómeno se denomina bloqueo. El bloqueo se da cuando el establecimiento previo de un elemento de una clave compuesta como señal del reforzador reduce o bloquea lo que se puede aprender acerca del segundo (Kamin, 1969). El Cuadro 1 resume los detalles principales del procedimiento. Existe una gran cantidad de trabajos que muestran la presencia del fenómeno de bloqueo en diferentes grupos de vertebrados e incluso invertebrados, bajo distintas condiciones de entrenamiento (ver Cuadro 2). En todos ellos se observa un fuerte bloqueo entre claves, sin importar la naturaleza de las mismas, ya que se ha observado con claves olfatorias, visuales, auditivas e incluso estructurales.

En algunas situaciones de entrenamiento donde se ofrecen dos claves se evidencia otro fenómeno, donde la clave más conspicua suele ensombrecer a la otra, quitándole poder de asociación con el reforzador. Surge entonces el término ensombrecimiento, que se refiere al hecho de que una segunda clave, muy conspicua y saliente, le quita poder asociativo a la primera, menos conspicua (Kamin, 1969; Pavlov, 1927). El Cuadro 1 también resume los detalles principales del procedimiento de ensombrecimiento. Muchos estudios (ver Cuadro 2) muestran este fenómeno en distintas condiciones de entrenamiento, utilizando diferentes modelos experimentales, desde caracoles terrestres hasta humanos (Chamizo, Sterio \& Mackintosh, 1985) y verificaron por primera vez la existencia de los fenómenos de bloqueo y ensombrecimiento usando claves visuales locales en una tarea de aprendizaje espacial en ratas. Estos trabajos permitieron luego desarrollar una serie de análisis de dichos fenómenos, en los cuales se pudo determinar que las claves espaciales que rodean a un laberinto de Morris pueden afectar al aprendizaje de esta tarea de modo similar a los estímulos condicionados en el condicionamiento clásico o a los estímulos discriminativos en el condicionamiento instrumental (Rodrigo, Chamizo, McLaren \& Mackintosh, 1997; Sánchez-Moreno, Rodrigo, Chamizo \& Mackintosh, 1999).

El efecto de bloqueo entre claves visuales en un contexto de aprendizaje espacial también ha sido reportado en humanos utilizando una versión computarizada del laberinto de Morris (Hamilton \& Sutherland, 1999). Los sujetos inicialmente entrenados para localizar una meta invisible con un determinado conjunto de claves, tuvieron un pobre desempeño al intentar localizar la meta cuando fueron probados con un grupo de claves agregadas con posterioridad (encontrándose un claro efecto de bloqueo).

El ensombrecimiento entre claves visuales ha sido descripto anteriormente por Spetch (1995), quien realizó un análisis comparado de experimentos en palomas y humanos utilizando una pantalla táctil y claves generadas por computadora. En este estudio se observó que en ambas especies, el control adquirido sobre la respuesta (picotear en las palomas y presionar en los humanos) por una clave a una distancia dada del objetivo se reducía en presencia de otra clave más cercana. Estos resultados son una clara demostración de ensombrecimiento debido a la proximidad espacial relativa de una clave visual frente a otra. Este fenómeno de ensombrecimiento también ha sido hallado en humanos utilizando un procedimiento de navegación virtual (Chamizo, 2002).

En nuestro laboratorio se realizó una serie de experimentos que permitió determinar por primera vez la existencia de los fenómenos de bloqueo y ensombrecimiento en anfibios, con un paradigma de aprendizaje espacial (Daneri \& Muzio, 2013). Los cuales se describen brevemente. Como es habitual en todos nuestros estudios en anfibios, en ambas situaciones se utilizaron machos adultos del sapo terrestre Rhinella arenarum parcialmente deshidratados (al $80 \%$ de su peso para motivar la búsqueda de agua). Los entrenamientos se desarrollaron en una arena circular de color 
blanco $(86 \mathrm{~cm}$ de diámetro y $100 \mathrm{~cm}$ de altura), usando agua deionizada como reforzador (ver Figura 1). En el aparato experimental se ubicaron cuatro piletas idénticas de acrílico color verde $(12 \times 10 \times 4 \mathrm{~cm})$ distribuidas en cruz contra las paredes laterales, donde sólo una permitía el acceso al refuerzo. En las paredes internas de la arena circular se ubicaron distintas claves visuales (de 10 x 10 $\mathrm{cm}$, realizadas en goma eva) para que los animales se orientaran. En todos estos estudios los animales tuvieron un entrenamiento que constaba de una sesión diaria con tres ensayos cada una, comenzando cada ensayo ubicados en el centro de la arena circular. La principal variable dependiente utilizada fue el porcentaje de respuestas correctas, aunque también se registraron otras, como el promedio de sesiones hasta alcanzar el criterio de aprendizaje y la latencia de recorrido.

En el experimento de bloqueo, en una primera etapa de entrenamiento (18 sesiones), el grupo experimental tenía la pileta reforzada señalada por una clave visual (rombo rojo o panel rayado) pegada sobre la pared de la arena circular a $10 \mathrm{~cm}$ sobre la pileta. En una segunda etapa (16 sesiones) se agregó otra clave visual (la clave a bloquear, rombo rojo o panel rayado, según lo que hubieran recibido en la primera etapa). A los sujetos del grupo control sólo se les administró esta segunda etapa, donde la pileta reforzada siempre estaba señalada por las dos claves visuales ubicadas sobre la pileta (rombo rojo y cuadrado rayado). Los resultados de los ensayos de prueba revelaron que en los animales del grupo experimental, el entrenamiento previo con una sola de las claves visuales bloqueó la asociación del refuerzo con la otra clave (reflejado por un menor porcentaje de respuestas correctas) cuando, en la segunda fase del experimento, ambas claves fueron presentadas simultáneamente señalando la posición del refuerzo. Este fenómeno no se observó en los animales del grupo control (sin exposición previa), donde cada una de las dos claves de presentación simultánea fue asociada exitosamente con el reforzador. Este resultado demuestra que el bloqueo entre claves visuales durante el aprendizaje espacial es un fenó- meno generalizado, presente en una amplia variedad de vertebrados, que incluye también a los anfibios (Daneri \& Muzio, 2013).

En el experimento de ensombrecimiento, los animales experimentales tenían la pileta reforzada señalada por las dos claves visuales pegadas sobre la pared blanca de la arena a $10 \mathrm{~cm}$ de altura: la primera, ubicada a $10 \mathrm{~cm}$ a la derecha de la pileta reforzada (denominada clave cercana) y la segunda, colocada aproximadamente a $70 \mathrm{~cm}$ a la izquierda sobre la pared que estaba entre la pileta contigua y la opuesta (denominada clave lejana). Los animales del grupo control tenían la pileta reforzada señalada por sólo una clave visual lejana (ubicada también a la izquierda, sobre la pared que estaba entre la pileta contigua y la opuesta a la pileta reforzada). Luego de 24 sesiones de adquisición, los resultados de los ensayos de prueba indicaron que la ubicación de una clave visual localizada lejos del refuerzo fue ensombrecida por la presencia de una clave cercana (en los animales del grupo experimental el estímulo lejano no adquirió ningún valor predictivo, mostrando un bajo porcentaje de respuestas correctas). Este fenómeno no se observó en los animales del grupo control, donde la clave lejana fue estrechamente asociada con la presencia del reforzador y pudo ser utilizada con éxito para predecir su posición. Estos resultados mostraron claramente que el fenómeno de ensombrecimiento también está presente en anfibios (Daneri \& Muzio, 2013).

Globalmente, el conjunto de resultados anteriores sugiere que el bloqueo y el ensombrecimiento entre claves son fenómenos presentes en un amplio grupo de seres vivos, incluyendo tanto vertebrados como invertebrados. El hallazgo de estos dos fenómenos de aprendizaje en anfibios se suma a los resultados anteriores y apoya la idea de un origen evolutivo temprano de los mismos.

\section{INHIBICIÓN LATENTE}

La inhibición latente es un fenómeno que se observa cuando la exposición previa a un estímulo, en ausencia de un reforzador, re- 
tarda significativamente el condicionamiento subsecuente de ese estímulo cuando se presenta seguido de un reforzador o un EI (Lubow, 1973). El Cuadro 1 también resume los detalles principales del procedimiento de inhibición latente. Actualmente, la interferencia generada por la pre-exposición no reforzada a un estímulo sobre el condicionamiento subsiguiente a ese estímulo está bien establecida y ha sido demostrada en una amplia variedad de especies y procedimientos (ver Cuadro 2). Sin embargo, existe menos consenso acerca de la explicación de este fenómeno (De la Casa \& Lubow, 2005; Mackintosh, 1974). La interpretación del fenómeno de inhibición latente puede verse desde dos puntos de vista: como una forma de habituación, o como una pérdida en el valor de predicción. En el primer caso, la presentación repetida de un estímulo puede reducir su novedad; el sujeto se familiariza con él y ya no se sorprende con su presentación. Visto en este sentido, la inhibición latente sería una forma de habituación. En el segundo caso, el estímulo se presenta repetidamente sin ser seguido por otro evento, perdiendo así el valor predictivo que puede tener un estímulo novedoso. Como el condicionamiento involucra el establecimiento de un estímulo como predictor de un evento, este cambio en el valor predictivo subyace a la baja asociabilidad del estímulo pre-expuesto (Mackintosh, 1983).

Con respecto a la presencia del fenómeno de inhibición latente en un contexto de aprendizaje espacial, existe evidencia previa en un trabajo de Chamizo y Mackintosh (1989) realizado con ratas y utilizando un laberinto elevado con distintos tipos de claves visuales tanto intra como extra laberinto. En esta situación pudo demostrarse que la pre-exposición no reforzada a uno de los brazos del laberinto que tenía sus paredes coloreadas retrasó la adquisición del aprendizaje una vez que se introdujo el reforzador.

Nuestro grupo de trabajo también ha llevado a cabo una serie de experimentos con el fin de establecer si el fenómeno de inhibición latente se observa en anfibios (Daneri, Casanave \& Muzio, 2015b). Al igual que en los experimentos de bloqueo y de ensombreci- miento, en estos estudios previos se utilizaron machos adultos del sapo terrestre Rhinella arenarum parcialmente deshidratados (al 80\% de su peso) y entrenados en una arena circular, usando agua como reforzador. Sobre las paredes internas de la arena se ubicaron distintas claves visuales cercanas para que los animales se orientaran. Los animales del grupo pre-expuesto tuvieron cinco sesiones previas al entrenamiento, en las que se presentaba una clave visual sin reforzador. Luego, cuando en el entrenamiento posterior esta clave visual señalaba la recompensa, al comparar el número promedio de sesiones hasta lograr el aprendizaje de este grupo pre-expuesto con otro grupo no pre-expuesto, se observó una diferencia significativa: los animales del grupo pre-expuesto necesitaron más entrenamiento para llegar a la asíntota de aprendizaje (aproximadamente 16 sesiones, en tanto que sólo 10 sesiones en promedio para el grupo sin pre-exposición). Por lo tanto, la pre-exposición a la clave visual (exposición no reforzada del estímulo) retrasó significativamente la adquisición, demorando la asociación de este estímulo con el reforzador.

Estos resultados revelan entonces por primera vez que los anfibios también presentan el fenómeno de inhibición latente usando claves visuales en una situación de aprendizaje espacial (Daneri et al., 2015b).

\section{DisCUSIÓN Y CONCLUSIONES}

En primer lugar, el conjunto de resultados obtenidos en nuestro laboratorio indica que por primera vez los fenómenos de bloqueo, ensombrecimiento e inhibición latente (previamente documentados en otros vertebrados e incluso en invertebrados), también están presentes en anfibios. Esta información confirma el paralelismo establecido entre el aprendizaje espacial mediante el uso de claves visuales proximales y otras formas de aprendizaje, las que estarían gobernadas por las mismas leyes asociativas. En este sentido, en términos generales, el conjunto de estos fenómenos (observados en una situación de aprendizaje espacial o no) comparten una 
dinámica asociativa común, ya que todos ellos son fenómenos proactivos de procesamiento de la información (i.e., memorias previas interfieren con la adquisición de nuevos aprendizajes) (Loy, Fernandez \& Acebes, 2006). Por lo tanto, es altamente probable que los procesos de aprendizaje asociativo observados en estos experimentos jueguen un papel importante en los procesos a través de los cuales un animal se orienta en el espacio.

Dado que estos fenómenos han sido observados previamente en aves y mamíferos, este primer registro en anfibios (empleando un paradigma de aprendizaje espacial con claves visuales cercanas y lejanas) sugiere que los mecanismos biológicos de estos fenómenos de aprendizaje habrían surgido muy tempranamente en el curso de la evolución de los vertebrados plenamente terrestres y que los mismos han sido fuertemente conservados. Por otra parte, debemos tener en cuenta que la evolución ha requerido del desarrollo exitoso de sistemas de defensa para afrontar los peligros que amenazaban con interrumpir la transmisión de genes (y la información codificada por los mismos) entre generaciones. El escape y la evitación han sido estrategias claves con alto valor adaptativo desarrolladas con este fin (Öhman \& Mineka, 2001). En este aspecto, el aprendizaje espacial es crucial para la supervivencia de una especie ya que contribuye no sólo a mejorar las conductas de escape y evitación de predadores, sino también a optimizar la ubicación de fuentes de alimento y agua, refugio y potenciales parejas. Por lo tanto, es esperable que este tipo de aprendizaje también haya sido moldeado por los procesos evolutivos (Bingman, Salas \& Rodríguez, 2009; Sotelo, Bingman \& Muzio, 2015).

Desde esta perspectiva, es muy probable que las relaciones entre claves ambientales y sus consecuencias relevantes para la supervivencia sean usadas por la selección natural para promover su asociación selectiva y preferencial en el cerebro de los animales (cf., Bolles, 1970; Seligman, 1970). Con la aparición de sistemas nerviosos más complejos, las habilidades seleccionadas por la evolución pudieron involucrar mecanismos más so- fisticados y selectivos (Öhman \& Mineka, 2001). Así, los fenómenos de bloqueo, ensombrecimiento e inhibición latente podrían haber sido seleccionados por procesos evolutivos con el fin de maximizar la eficiencia del uso de claves ambientales y particularmente, en los procedimientos descriptos en este artículo, con el objetivo de optimizar el uso de claves visuales ambientales para la orientación espacial.

Finalmente, si se consideran los sustratos neurales implicados, la relación de la formación hipocampal con los procesos de aprendizaje y memoria espacial aparece como una constante en todas las especies de vertebrados estudiadas (Rodríguez, López, Vargas \& Salas, 1998). Analizando el caso particular de los anfibios, en comparación con los demás grupos de tetrápodos, su telencéfalo presenta una organización simple (en el sentido no derivado u original, definido en términos evolutivos). Así, el telencéfalo de los anfibios puede dividirse en una región palial (palio o pallium) y otra subpalial (Muzio, 1999, 2013; Rodríguez et al., 1998). En el palio de los anfibios pueden distinguirse tres zonas principales: (1) el pallium medial, considerado homólogo a la formación hipocampal de los mamíferos, que es la región más extensa del palio y ocupa el cuadrante dorsomedial del hemisferio, (2) el pallium dorsal, que puede considerarse homólogo al isocortex de otros vertebrados y se continúa lateralmente con el pallium medial, sin que se aprecie una frontera precisa entre ambas zonas y (3) el pallium lateral, que es considerado homólogo al córtex piriforme de otros vertebrados y ocupa el cuadrante dorsolateral del hemisferio (Muzio, 2013; Northcutt \& Kicliter, 1980).

La hipótesis muy aceptada respecto de que el pallium medial de los anuros es homólogo al complejo hipocampal de otros tetrápodos, está basada en su posición topográfica (cuadrante dorsomedial telencefálico), derivada de un desarrollo cerebral por evaginación (Papini, Salas \& Muzio, 1999), y de sus interconexiones neurales con otras áreas telencefálicas, así como de datos histoquímicos (Northcutt \& Ronan, 1992). Todas estas características convierten al pallium medial en la estructura de 
mayor interés en el estudio del aprendizaje espacial en anfibios (Muzio, 2013).

En relación a los aspectos neuromorfológicos detallados anteriormente, cabe mencionar que en términos generales los amniotas (reptiles, aves y mamíferos) son considerados como un grupo monofilético que evolucionó de un conjunto primitivo de tetrápodos durante los inicios del Carbonífero (Carroll, 1988; Gaffney, 1980). De esta manera, el hallazgo en reptiles, aves y mamíferos de que las capacidades de memoria y la presencia de aprendizaje espacial alocéntrico (es decir, usando claves visuales externas) eran dependientes de la corteza medial, parecía indicar que este rasgo podría ser un carácter primitivo de los amniotas. Así, estas capacidades de aprendizaje podrían ya haber estado presentes en el ancestro común reptiliano de los amniotas modernos que habitó la tierra en la era Mesozoica, siendo conservadas a lo largo de la evolución en cada linaje en forma independiente (Salas, Broglio \& Rodríguez, 2003). Sin embargo, la dependencia del aprendizaje espacial con el funcionamiento de la corteza medial puede extenderse ahora al grupo de los anfibios, ya que recientemente se ha observado que la lesión del pallium medial deteriora el aprendizaje espacial (Daneri, Casanave \& Muzio, 2015c). De esta forma, la evidencia reunida hasta este momento en anfibios sugiere que esta relación pudo haber surgido más tempranamente de lo que se pensaba.

En síntesis, la información obtenida a partir de nuestros estudios en anfibios constituye un avance importante en el análisis comparado de estos fenómenos de aprendizaje, incorporando por primera vez a este grupo preamniota al conjunto de clases de vertebrados que tienen estas capacidades cognitivas.

\section{FIGURA 1}

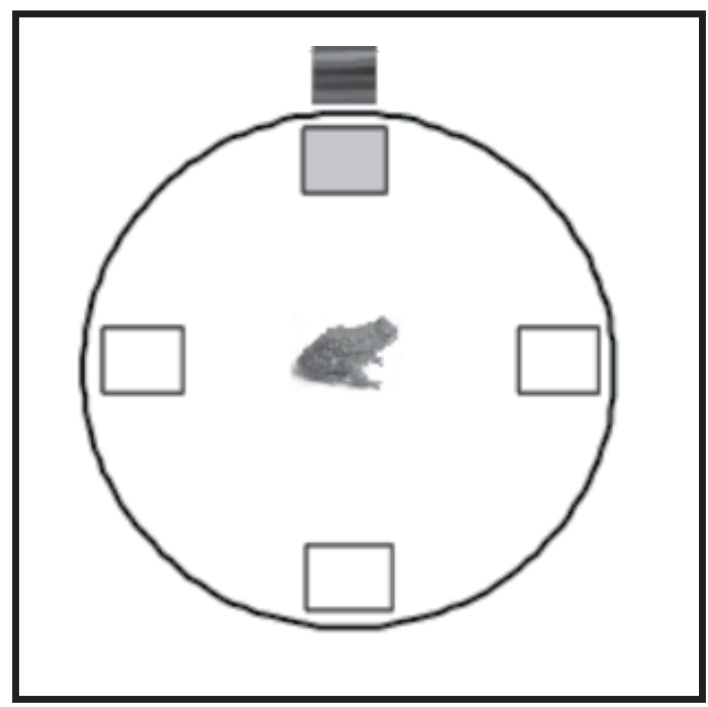




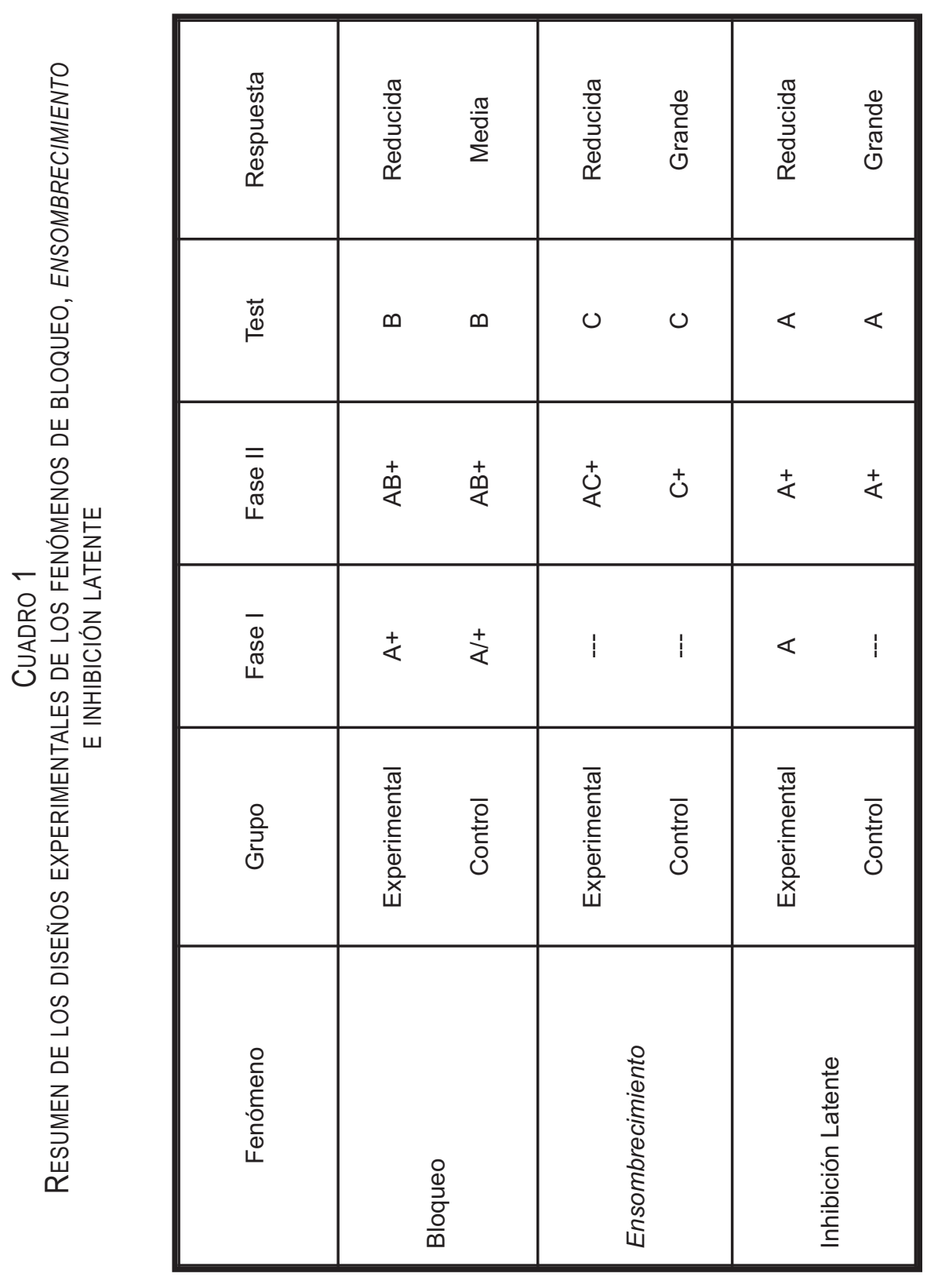

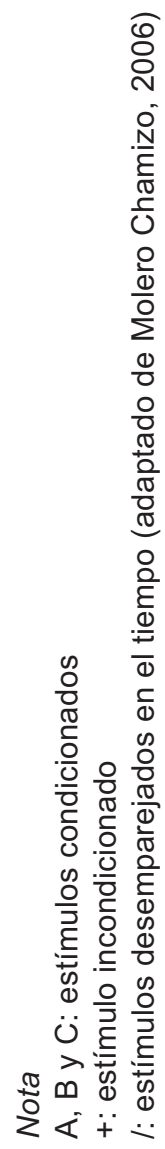


CUADRO 2

EJEMPLOS EXPERIMENTALES DE LOS FENÓMENOS DE BLOQUEO, ENSOMBRECIMIENTO E INHIBICIÓN LATENTE EN DIFERENTES ESPECIES DE VERTEBRADOS E INVERTEBRADOS

\begin{tabular}{|c|c|c|c|}
\hline Modelo & Bloqueo & Ensombrecimiento & Inhibición latente \\
\hline $\begin{array}{l}\text { Caracol } \\
\text { terrestre }\end{array}$ & $\begin{array}{l}\text { Claves olfativas en asociación } \\
\text { con alimento (Acebes et al., } \\
\text { 2009). }\end{array}$ & $\begin{array}{l}\text { Claves olfativas en asociación } \\
\text { con alimento (Loy et al., 2006). }\end{array}$ & $\begin{array}{l}\text { Claves olfativas en asociación } \\
\text { con alimento (Loy et al., 2006). }\end{array}$ \\
\hline Abejas & $\begin{array}{l}\text { Extensión de proboscis con } \\
\text { claves de olor (Hammer \& } \\
\text { Menzel, 1995). } \\
\text { Localización de alimento } \\
\text { utilizando claves de olor } \\
\text { (Bitterman, 1996). } \\
\text { Claves visuales en localización } \\
\text { de solución azucarada (Cheng } \\
\text { \& Spetch, 2001). }\end{array}$ & $\begin{array}{l}\text { Localización de alimento utili- } \\
\text { zando claves de color y olor } \\
\text { (Bitterman, 1996). }\end{array}$ & $\begin{array}{l}\text { Condicionamiento aversivo en } \\
\text { un contexto de alimentación } \\
\text { (Abramson \& Bitterman, 1986). }\end{array}$ \\
\hline Peces & $\begin{array}{l}\text { Orientación utilizando claves } \\
\text { de ángulo - color (Tennant \& } \\
\text { Bitterman, 1975). }\end{array}$ & $\begin{array}{l}\text { Orientación utilizando claves } \\
\text { de color-sonido y ángulo-color } \\
\text { (Tennant \& Bitterman, 1975). }\end{array}$ & $\begin{array}{l}\text { Claves olfativas para reconoci- } \\
\text { miento de predador (Ferrari \& Chi- } \\
\text { vers, 2006; Mitchell et al., 2011). }\end{array}$ \\
\hline Anfibios & $\begin{array}{l}\text { Orientación espacial en un } \\
\text { campo abierto utilizando } \\
\text { claves visuales intralaberinto } \\
\text { (Daneri \& Muzio, 2013). }\end{array}$ & $\begin{array}{l}\text { Orientación espacial en un } \\
\text { campo abierto utilizando } \\
\text { claves visuales intralaberinto } \\
\text { (Daneri \& Muzio, 2013). }\end{array}$ & $\begin{array}{l}\text { Orientación espacial en un } \\
\text { campo abierto utilizando } \\
\text { claves visuales intralaberinto } \\
\text { (Daneri et al., 2015b). }\end{array}$ \\
\hline Palomas & $\begin{array}{l}\text { Discriminación utilizando claves } \\
\text { con patrones de rayas y color } \\
\text { (Mackintosh \& Honig, 1970). }\end{array}$ & $\begin{array}{l}\text { Claves visuales en pantalla } \\
\text { táctil de computadora (Spetch, } \\
\text { 1995). }\end{array}$ & $\begin{array}{l}\text { Picoteo espontáneo de tecla } \\
\text { luminosa (Tranberg \& Rilling, } \\
\text { 1978). }\end{array}$ \\
\hline Ratas & $\begin{array}{l}\text { Claves visuales en laberinto } \\
\text { de Morris (Chamizo et al., } \\
\text { 1985. Roberts \& Pearce, 1999). } \\
\text { Forma de la pileta y color de } \\
\text { las paredes (Pearce et al., } \\
\text { 2006). } \\
\text { Bloques de madera en campo } \\
\text { abierto (Stahlman \& Blaisdell, } \\
\text { 2009). }\end{array}$ & $\begin{array}{l}\text { Claves visuales en laberinto } \\
\text { de Morris (Chamizo et al., } \\
\text { 1985). } \\
\text { Forma de la pileta y color de } \\
\text { las paredes (Pearce et al., } \\
\text { 2006). }\end{array}$ & $\begin{array}{l}\text { Uso de claves visuales intra y } \\
\text { extra laberinto (Chamizo \& } \\
\text { Mackintosh, 1989). } \\
\text { Aversión gustativa (De la Casa } \\
\text { \& Lubow, 2005). }\end{array}$ \\
\hline Humanos & $\begin{array}{l}\text { Claves visuales en navegación } \\
\text { virtual de laberinto (Hamilton \& } \\
\text { Sutherland, 1999). }\end{array}$ & $\begin{array}{l}\text { Claves visuales en pantalla } \\
\text { táctil de computadora (Spetch, } \\
\text { 1995). } \\
\text { Claves visuales en navegación } \\
\text { virtual de laberinto (Chamizo, } \\
\text { 2002). }\end{array}$ & $\begin{array}{l}\text { Inhibición de la respuesta de } \\
\text { parpadeo (Schnur \& Ksir, 1969). } \\
\text { Asociado a rasgos de personali- } \\
\text { dad (Carson et al., 2003). } \\
\text { Asociado a esquizofrenia (Lubow } \\
\& \text { Gewirtz, 1995). }\end{array}$ \\
\hline
\end{tabular}




\section{REFERENCIAS BIBLIOGRÁFICAS}

Abramson, C.I. \& Bitterman, M.E. (1986). Latent inhibition in honeybees. Animal Learning \& Behavior, 14, 184-189. http://dx.doi.org/10. 3758/bf03200054

Acebes, F., Solar, P., Carnero, S. \& Loy, I. (2009). Blocking of conditioning of tentacle lowering in the snail (Helix aspersa). Quarterly Journal of Experimental Psychology, 62(7), 13151327. http://dx.doi.org/10.1080/1747021080 2483545

Bingman, V.P., Salas, C. \& Rodríguez, F. (2009). Evolution of the hippocampus. En M. Binder, \& U. Windhorst (Eds.), Encyclopaedia of Neuroscience (pp. 1356-1360). Berlín: SpringerVerlag.

Bitterman, M.E. (1996). Comparative analysis of learning in honeybees. Animal Learning \& Behavior, 24(2), 123-141. http://dx.doi.org/10. 3758/bf03198961

Bolles, R.C. (1970). Species-specific defense reactions and avoidance learning. Psychological Review, 77, 32-48. http://dx.doi.org/10.1037/ h0028589

Carroll, R. (1988). Vertebrate paleontology and evolution. New York: Freeman. http://dx.doi. org/10.1080/02724634.1988.10 01170.

Carson, S.H., Peterson, J.B. \& Higgins, D.M. (2003). Decreased latent inhibition is associated with increased creative achievement in high-functioning individuals. Journal of Personality and Social Psychology, 85(3), 499506. http://dx.doi.org/10.1037/0022-3514.85. 3.499

Chamizo, V.D. (2002). Spatial learning: Conditions and basic effects. Psicológica, 23, 33-57. Recuperado el 25 de enero de 2012 de http:// www.uv.es/revispsi/articulos 1.02/M2Chami zo.pdf

Chamizo, V.D. \& Mackintosh, N.J. (1989). Latent learning and latent inhibition in maze discriminations. Quarterly Journal of Experi- mental Psychology, 41B, 21-31. http://dx.doi. org/10.1080/14640748908401181

Chamizo, V.D., Sterio, D. \& Mackintosh, N.J. (1985). Blocking and overshadowing between intra-maze and extra-maze cues: A test of the independence of locale and guidance learning. Quarterly Journal of Experimental Psychology, 37B, 235-253. http://dx.doi.org/10.1080/ 14640748508402098

Cheng, K. \& Spetch, M.L. (2001). Blocking in the spatial domain in honeybees. Animal Learning \& Behavior, 29, 1-9. http://dx.doi.org/10.3758 /bf03192811

Daneri, M.F., Casanave, E. \& Muzio, R.N. (2011). Control of spatial orientation in terrestrial toads (Rhinella arenarum). Journal of Comparative Psychology, 125(3), 296-307. http://dx.doi.org/10.1037/a0024242

Daneri, M.F., Casanave, E.B. \& Muzio, R.N. (2015a). Use of local visual landmarks for spatial orientation in toads (Rhinella arenarum): The role of distance to a goal. Journal of Comparative Psychology. http://dx.doi.org/10.103 7/a0039461

Daneri, M.F., Casanave, E.B. \& Muzio, R.N. (2015b). Blocking, overshadowing and latent inhibition in terrestrial toads (Rhinella arenarum): Use of visual cues for orientation. Trabajo inédito.

Daneri, M.F., Casanave, E.B. \& Muzio, R.N. (2015c). Medial Pallium lesion affects both turn and cue spatial learning in terrestrial toads (Rhinella arenarum). Trabajo inédito.

Daneri, M.F. \& Muzio, R.N. (2013). Bloqueo y ensombrecimiento en un grupo de vertebrados filogenéticamente antiguo: Los anfibios [Blocking and overshadowing in a phylogenetically ancient vertebrate group: Amphibians]. Revista Latinoamericana de Psicología, 45(2), 185200. http://dx.doi.org10.14349/rlp.v4 5i 2.1138

Day, L.B., Ismail, N. \& Wilczynski, W. (2003). Use of position and feature cues in discrimination learning by the Wiptail Lizard (Cnemi- 
dophorus inornatus). Journal of Comparative Psychology, 117(4), 440-448. http://dx.doi. org/10.1037/0735-7036.117.4.440

De la Casa, L.G. \& Lubow, R.E. (2005). Delay-induced super-latent inhibition as a function of order of exposure to two flavours prior to compound conditioning. Quarterly Journal of Experimental Psychology, 58B, 1-18. Recuperado el 20 de noviembre de 2014 de http://ww w.ncbi.nlm.nih.gov/pubmed/1584 4374\#

Ferrari, M.C.O. \& Chivers, D.P. (2006). The role of latent inhibition in acquired predator recognition by fathead minnows. Canadian Journal of Zoology, 84, 505-509. http://dx.doi.org/10. 1139/z06-027

Freeland, W.J. \& Martin, K.C. (1985). The rate of range expansion by Bufo marinus in Northern Australia, 1980-84. Wildlife Research, 12(3), 555-559. http://dx.doi.org/10.1071/wr9850555

Gaffney, E.S. (1980). Phylogenetic relationships of the major groups of amniotes. En A.L. Panchen (Ed.), The terrestrial environment and the origin of land vertebrates (pp. 593610). London: Academic Press.

Gallardo, J.M. (1987). Anfibios argentinos: Guía para su identificación [Argentinean amphibians: Guide for their identification]. Buenos Aires: Librería Agropecuaria.

Grisham, W. \& Powers, A. (1990). Effects of dorsal and medial cortex lesions on Rreversal in turtles. Physiology and Behavior, 47, 43-49. http://dx.doi.org/10.1016/0031-9384(90)90 040-B

Hamilton, D.A. \& Sutherland, R.J. (1999). Blocking in human place learning: Evidence from virtual navigation. Psychobiology, 27, 453461. http://dx.doi.org/10.3758/BF03332140

Hammer, M. \& Menzel, R. (1995). Learning and memory in the honeybee. The Journal of Neuroscience, 15(3), 1617-1630. http://dx.doi.org/ 10.1007/bf01143197

Kamin, L.J. (1969). Predictability, surprise, attention and conditioning. En B.A. Campbell \&
R.M. Church(Eds.), Punishment and aversive behavior. NY: Appleton-Century-Crofts.

López, J.C., Broglio, C, Rodríguez, F, ThinusBlanc, C. \& Salas, C. (1999). Multiple spatial learning strategies in goldfish (Carassius auratus). Animal Cognition, 2, 109-120. http:// dx.doi.org/10.1007/s100710050031

López, J.C., Vargas, J.P., Gómez, Y. \& Salas, C. (2003). Spatial and non-spatial learning in turtles: The role of medial cortex. Behavioral Brain Research, 143, 109-120. http://dx.doi. org/10.1016/S0166-4328(03)00030-5

Loy, I., Fernandez, V. \& Acebes, F. (2006). Conditioning of tentacle lowering in the snail ( $\mathrm{He}$ lix aspersa): Acquisition, latent inhibition, overshadowing, second-order conditioning and sensory preconditioning. Learning and Behavior, 34(3), 305-314. http://dx.doi.org/1 0.3758/BF03192885

Lubow, R.E. (1973). Latent inhibition. Psychological Bulletin, 79, 398-407. http://dx.doi.or g/10.1037/h0034425

Lubow, R.E. \& Gewirtz, J.C. (1995). Latent inhibition in humans: Data, theory, and implications for schizophrenia. Psychological Bulletin, 117(1), 87-103. http://dx.doi.org/10.103 7/0033-2909.117.1.87

Lüddecke, H. (2003). Space use, cave choice and spatial learning in the dendrobatid frog Colostethus palmatus. Amphibia-Reptilia, 24, 37-46. http://dx.doi.org/10.1163/15685380 3763806920

Mackintosh, N.J. (1974). The psychology of learning. London: Academic Press.

Mackintosh, N.J. (1983). Conditioning and associative learning. London: Academic Press.

Mackintosh, N.J. \& Honig, W.K. (1970). Blocking and enhancement of stimulus control in pigeons. Journal of Comparative and Physiological Psychology, 73(1), 78-85. http://dx.do i.org/10.1037/h0030021

Mitchell, M.D., McCormick, M.I., Ferrari, M. C.O. \& Chivers, D.P. (2011). Friend or foe? 
The role of latent inhibition in predator and non-predator labelling by coral reef fishes. Animal Cognition, 14(5), 707-714. http://dx. doi.org/10.1007/s10071-011-0405-6

Molero Chamizo, A. (2006). Características experimentales y neurobiología de la inhibición latente en el paradigma de aprendizaje aversivo gustativo [Experimental characteristics and neurobiology of latent inhibition in an aversive gustative learning paradigm] Psicológica, 27, 169-194. Recuperado el 13 de mayo de 2015 de https://www.uv.es/psicologica/articulos 2.06/2 Molero.pdf

Muzio, R.N. (1999). Aprendizaje instrumental en anfibios [Instrumental learning in amphibians]. Revista Latinoamericana de Psicología, 31, 35-47. Recuperado el 19 de marzo de 2010 de http://www.redalyc.org/articulo.oa? id $=80531103$

Muzio, R.N. (2013). Aprendizaje en anfibios, el eslabón perdido: Un modelo simple cerebral en el estudio de conductas complejas [Learning amphibians, the missing link: A simple model to study brain complex behaviors]. Cuadernos de Herpetología, 27(2), 87-100. Recuperado el 11 de agosto de 2014 de http:// ppct.caicyt.gov.ar/index.php/cuadherpetol/article/view/1365/2337

Northcutt, R.G. \& Kicliter, E. (1980). Organization of the amphibian telencephalon. En S. O.E. Ebbesson (Ed.), Comparative neurology of the telencephalon (pp. 203-255). New York: Plenum.

Northcutt, R.G. \& Ronan, M. (1992). Afferent and efferent connections of the bullfrog medial pallium. Brain, Behavior and Evolution, 40, 116. http://dx.doi.org/10.1159/000113898

Öhman, A. \& Mineka, S. (2001). Fears, phobias, and preparedness: Toward an evolved module of fear and fear learning. Psychological Review, 108(3), 483-522. http://dx.doi.org/10.1 037//0033-295x.108.3.483

Papini, M.R. (2002). Pattern and process in the evolution of learning. Psychological Review,
109, 186-201. http://dx.doi.org/10.1037/0033295X.109.1.186

Papini, M.R. (2009). Psicología comparada. Evolución y desarrollo del comportamiento [Comparative Psychology. Evolution and development of behavior] (R.N. Muzio et al. Trads.). Bogotá, Colombia: Editorial Manual Moderno. (Trabajo original publicado en 2002).

Papini, M.R., Salas, C. \& Muzio, R.N. (1999). Análisis comparativo del aprendizaje en vertebrados [Comparative analysis of learning in vertebrates]. Revista Latinoamericana de Psicología, 31, 15-34. Recuperado el 19 de marzo de 2010 de http://www.redalyc.org/articulo.o $\mathrm{a} ? \mathrm{id}=80531102$

Pavlov, I.P. (1927). Conditioned reflexes. Londres: Oxford University Press.

Pearce, J.M., Graham, M., Good, M.A., Jones, P.M. \& McGregor, A. (2006). Potentiation, overshadowing, and blocking of spatial learning based on the shape of the environment. Journal of Experimental Psychology: Animal Behavior Processes, 32(3), 201-214. http://dx. doi.org/10.1037/0097-7403.32.3.201

Roberts, A.D.L. \& Pearce, J.M. (1999). Blocking in the Morris swimming pool. Journal of Experimental Psychology: Animal Behavior Processes, 25(2), 225-235. http://dx.doi.org/10. 1037//0097-7403.25.2.225

Rodrigo, T., Chamizo, V.D., McLaren, I.P.L. \& Mackintosh, N.J. (1997). Blocking in the spatial domain. Journal of Experimental Psychology: Animal Behavior Processes, 23, 110-118. http:// dx.doi.org/doi/10.1037/0097-7403.23.1.110

Rodríguez, F., López, J.C., Vargas, J.P. \& Salas, C. (1998). Fundamentos de psicobiología [Psychobiology foundations]. Sevilla: Kronos.

Salas, C., Broglio, C. \& Rodríguez, F. (2003). Evolution of forebrain and spatial cognition in vertebrates: Conservation across diversity. Brain, Behavior and Evolution, 62, 72-82. http://dx. doi.org/10.1159/000072438

Sánchez-Moreno, J., Rodrigo, T, Chamizo, V.D. \& Mackintosh, N.J. (1999). Overshadowing in 
the spatial domain. Animal Learning and Behavior, 27, 391-398. http://dx.doi.org/doi/10. 3758/BF03209976

Schnur, P. \& Ksir, C.J. (1969). Latent inhibition in human eyelid conditioning. Journal of Experimental Psychology, 80, 388-389. http://dx. doi.org/10.1037/h0027257

Seligman, M.E.P. (1970). On the generality of the laws of learning. Psychological Review, 77, 406-418. http://dx.doi.org/10.1037/h0029790

Sherry, D.F. \& Schacter, D.L. (1987). The evolution of multiple memory systems. Psychological Reviews, 194, 439-454. http://dx.doi.org/ doi/10.1037/0033-295X.94.4.439

Sinsch, U. (1987). Orientation behaviour of toads (Bufo bufo) displaced from the breeding site. Journal of Comparative Physiology A, 161(5), 715-727. http://dx.doi.org/10.1007/bf006050 13

Sinsch, U. (2006). Orientation and navigation in Amphibia. Marine and Freshwater Behavior and Physiology, 39(1), 65-71. http://dx.doi. org/doi/10.1080/10236240600562794

Sotelo, M.I., Bingman, V.P. \& Muzio, R.N. (2015). Goal orientation by geometric and feature cues: Spatial learning in the terrestrial toad Rhinella arenarum. Animal Cognition, 18(1), 315-323. http://dx.doi.org/10.1007 s100 71-014-0802-8.
Spetch, M.L. (1995). Overshadowing in landmark learning: Touch-screen studies with pigeon and humans. Journal of Experimental Psychology: Animal Behavior Processes, 21, 166-181. http://dx.doi.org/doi/10.1037/00977403.21.2.166

Stahlman, W.D. \& Blaisdell, A.P. (2009). Blocking of spatial control by landmarks in rats. Behavioral Processes, 81(1), 114-118. http:// dx.doi.org/doi/10.1016/j.beproc.2009.02.007

Tennant, (1975). Blocking and overshadowing in two species of fish. Journal of Experimental Psychology: Animal Behavior Processes, 1(1), 22-29. http://dx.doi.org/10.1037/0097-7403. 1.1 .22

Tranberg, D.K. \& Rilling, M. (1978). Latent inhibition in the autoshaping paradigm. Bulletin of the Psychonomic Society, 11(5), 273-276. http://dx.doi.org/10.3758/bf03336828

Tunner, H.G. (1992). Locomotory behaviour in water frogs from Neusiedlersee (Austria, Hungary). $15 \mathrm{~km}$ migration of Rana lessonae and its hybridogenetic associate Rana esculenta. Proceedings of the 6th Ordinary General Meeting SEH, Budapest (pp. 449-452). Budapest, Hungary: Hungarian Natural History Museum. 